\title{
Matching heavy-quark fields in QCD and HQET at three loops
}

\author{
A.G. Grozin \\ Institut für Theoretische Teilchenphysik, Karlsruher Institut für Technologie, \\ 76128 Karlsruhe, Germany
}

\begin{abstract}
The relation between the heavy-quark field in QCD and the corresponding field in HQET is derived up to three loops, and to all orders in the large- $\beta_{0}$ limit. The corresponding relation between the QED electron field and the Bloch-Nordsieck one is gauge invariant to all orders. We also prove that the $\overline{\mathrm{MS}}$ anomalous dimension of the QED electron field depends on the gauge parameter only at one loop.
\end{abstract}

QCD problems with a single heavy quark $Q$ can be treated in a simpler effective theory - HQET, if there exists a 4-velocity $v$ such that the heavy-quark momentum is $p=m v+k$ ( $m$ is the on-shell mass) and the characteristic residual momentum is small: $k \ll m$. QCD operators can be written as series in $1 / m$ via HQET operators; the coefficients in these series are determined by matching on-shell matrix elements in both theories. For example, the heavylight quark currents have been considered at the leading (zeroth) order in $1 / \mathrm{m}$ up to three-loop accuracy [12].

Here we shall consider the heavy-quark field $Q$. Though its matrix elements are not directly observable, its matching coefficient can have some applications. For example, it is not possible to simulate heavy quarks on a lattice directly, because at present we cannot have lattice spacings $a \ll 1 / m$. On the other hand, simulating HQET on a lattice only requires $a \ll 1 / \Lambda_{\overline{\mathrm{MS}}}$. It is possible to obtain the HQET heavy-quark propagator in the Landau gauge from such simulations. Then, if we know the matching coefficient, we can reconstruct a fundamental QCD quantity - the heavy-quark propagator as a function of $x$.

At the tree level, $Q$ is related to the corresponding HQET field $Q_{v}$ (satisfying $\left.\psi Q_{v}=Q_{v}\right)$ by $3,4,5,6$

$$
Q(x)=e^{-i m v \cdot x}\left(1+\frac{i \not D_{\perp}}{2 m}+\cdots\right) Q_{v}(x), \quad D_{\perp}^{\mu}=D^{\mu}-v^{\mu} v \cdot D .
$$


The matrix elements of the bare fields between the on-shell quark with momentum $p=m v+k$ and the vacuum in both theories are given by the on-shell wave-function renormalization constants:

$$
<0\left|Q_{0}\right| Q(p)>=\left(Z_{Q}^{\mathrm{os}}\right)^{1 / 2} u(p), \quad<0\left|Q_{v 0}\right| Q(p)>=\left(\tilde{Z}_{Q}^{\mathrm{os}}\right)^{1 / 2} u_{v}(k)
$$

(HQET renormalization constants are denoted by $\tilde{Z}$ ). The Dirac spinors are related by the Foldy-Wouthuysen transformation

$$
u(m v+k)=\left[1+\frac{\not k}{2 m}+\mathcal{O}\left(\frac{k^{2}}{m^{2}}\right)\right] u_{v}(k) .
$$

Therefore, the bare fields are related by

$$
Q_{0}(x)=e^{-i m v \cdot x}\left[z_{0}^{1 / 2}\left(1+\frac{i \not D_{\perp}}{2 m}\right) Q_{v 0}(x)+\mathcal{O}\left(\frac{1}{m^{2}}\right)\right]
$$

where the bare matching coefficient is

$$
z_{0}=\frac{Z_{Q}^{\mathrm{os}}\left(g_{0}^{\left(n_{l}+1\right)}, a_{0}^{\left(n_{l}+1\right)}\right)}{\tilde{Z}_{Q}^{\mathrm{os}}\left(g_{0}^{\left(n_{l}\right)}, a_{0}^{\left(n_{l}\right)}\right)}
$$

(we use the covariant gauge: the gauge-fixing term in the Lagrangian is $-\left(\partial_{\mu} A_{0}^{a \mu}\right) /\left(2 a_{0}\right)$, and the free gluon propagator is $\left(-i / p^{2}\right)\left(g_{\mu \nu}-(1-\right.$ $\left.\left.a_{0}\right) p_{\mu} p_{\nu} / p^{2}\right)$; the number of flavours in QCD is $\left.n_{f}=n_{l}+1\right)$. The $\mathcal{O}(1 / m)$ matching coefficient in (3) is equal to the leading one, $z_{0}$; this reflexes the reparametrization invariance [7]. The $\overline{\mathrm{MS}}$ renormalized fields are related by the formula similar to (3), with the renormalized decoupling coefficient

$$
z(\mu)=\frac{\tilde{Z}_{Q}\left(\alpha_{s}^{\left(n_{l}\right)}(\mu), a^{\left(n_{l}\right)}(\mu)\right)}{Z_{Q}\left(\alpha_{s}^{\left(n_{l}+1\right)}(\mu), a^{\left(n_{l}+1\right)}(\mu)\right)} z_{0}
$$

If there are no massive flavours except $Q$, then $\tilde{Z}_{Q}^{\text {os }}=1$ because all loop corrections are scale-free. The QCD on-shell renormalization constant $Z_{Q}^{\mathrm{os}}$ contains the single scale $m$ in this case; it has been calculated [8] up to three loops. The three-loop $\overline{\mathrm{MS}}$ anomalous dimensions of $Q_{v}, 8,9$ and $Q$ [10,11 are also known. We have to express all three quantities $Z_{Q}^{\mathrm{os}}\left(g_{0}^{\left(n_{l}+1\right)}, a_{0}^{\left(n_{l}+1\right)}\right)$, $Z_{Q}\left(\alpha_{s}^{\left(n_{l}+1\right)}(\mu), a^{\left(n_{l}+1\right)}(\mu)\right), \tilde{Z}_{Q}\left(\alpha_{s}^{\left(n_{l}\right)}(\mu), a^{\left(n_{l}\right)}(\mu)\right)$ via the same variables, say, $\alpha_{s}^{\left(n_{l}\right)}(\mu), a^{\left(n_{l}\right)}(\mu)$, see [12]. An explicit formula expressing $\alpha_{s}^{\left(n_{l}+1\right)}(\mu)$ via $\alpha_{s}^{\left(n_{l}\right)}(\mu)$ and $L=2 \log (\mu / m)$ ( $m$ is the on-shell mass) can be found in [13]. The corre- 
sponding relation between $a^{\left(n_{l}+1\right)}(\mu)$ and $a^{\left(n_{l}\right)}(\mu)$ is

$$
\begin{aligned}
& \frac{a^{\left(n_{l}+1\right)}(\mu)}{a^{\left(n_{l}\right)}(\mu)}=1-\left[\frac{4}{3} L+\frac{6 L^{2}+\pi^{2}}{9} \varepsilon+\frac{2 L^{3}+\pi^{2} L-4 \zeta_{3}}{9} \varepsilon^{2}+\cdots\right] T_{F} \frac{\alpha_{s}^{\left(n_{l}\right)}(\mu)}{4 \pi} \\
& -\left[C_{A} L^{2}+\left(4 C_{F}+5 C_{A}\right) L+15 C_{F}-\frac{13}{12} C_{A}\right. \\
& \quad+\left(C_{A} L^{3}+\left(4 C_{F}+5 C_{A}\right) L^{2}+\left(30 C_{F}+\frac{\pi^{2}-13}{6} C_{A}\right) L\right. \\
& \left.\left.\quad+\left(\frac{\pi^{2}}{3}+\frac{31}{2}\right) C_{F}+\frac{5 \pi^{2}+169}{12} C_{A}\right) \varepsilon+\cdots\right] T_{F}\left(\frac{\alpha_{s}^{\left(n_{l}\right)}(\mu)}{4 \pi}\right)^{2}+\cdots
\end{aligned}
$$

Our main result is the renormalized matching coefficient

$$
\begin{aligned}
z(\mu)= & -(3 L+4) C_{F} \frac{\alpha_{s}^{\left(n_{l}\right)}(\mu)}{4 \pi}+\left(z_{22} L^{2}+z_{21} L+z_{20}\right) C_{F}\left(\frac{\alpha_{s}^{\left(n_{l}\right)}(\mu)}{4 \pi}\right)^{2} \\
& +\left(z_{33} L^{3}+z_{32} L^{2}+z_{31} L+z_{30}\right) C_{F}\left(\frac{\alpha_{s}^{\left(n_{l}\right)}(\mu)}{4 \pi}\right)^{3}+\cdots
\end{aligned}
$$

where

$$
\begin{aligned}
z_{22}= & \frac{9}{2} C_{F}-\frac{11}{2} C_{A}+2 T_{F} n_{l}, \\
z_{21}= & \frac{27}{2} C_{F}-\frac{215}{6} C_{A}+\frac{38}{3} T_{F} n_{l}+2 T_{F}, \\
z_{20}= & \left(16 \pi^{2} \log 2-24 \zeta_{3}-13 \pi^{2}+\frac{241}{8}\right) C_{F} \\
& +\left(-8 \pi^{2} \log 2+12 \zeta_{3}+5 \pi^{2}-\frac{1705}{24}\right) C_{A} \\
& +\left(\frac{4}{3} \pi^{2}+\frac{113}{6}\right) T_{F} n_{l}+\left(-\frac{16}{3} \pi^{2}+\frac{947}{18}\right) T_{F}, \\
z_{33}= & -\frac{9}{2} C_{F}^{2}+\frac{33}{2} C_{F} C_{A}-\frac{121}{9} C_{A}^{2}-6 C_{F} T_{F} n_{l} \\
& +\frac{88}{9} C_{A} T_{F} n_{l}+\frac{1}{3} a^{\left(n_{l}\right)}(\mu) C_{A} T_{F}-\frac{16}{9} T_{F}^{2} n_{l}^{2}, \\
z_{32}= & -\frac{45}{2} C_{F}^{2}+135 C_{F} C_{A}-\frac{2671}{18} C_{A}^{2}-42 C_{F} T_{F} n_{l}-4 C_{F} T_{F} \\
& +\frac{938}{9} C_{A} T_{F} n_{l}-\left(\frac{13}{6} a^{\left(n_{l}\right)}(\mu)+1\right) C_{A} T_{F}-\frac{152}{9} T_{F}^{2} n_{l}^{2}+\frac{8}{3} T_{F}^{2}, \\
z_{31}= & \left.-48 \pi^{2} \log 2+72 \zeta_{3}+39 \pi^{2}-\frac{783}{8}\right] C_{F}^{2} \\
& +\left[\frac{424}{3} \pi^{2} \log 2-224 \zeta_{3}-\frac{331}{3} \pi^{2}+\frac{13307}{24}\right] C_{F} C_{A}
\end{aligned}
$$




$$
\begin{aligned}
& +\left[\left(-\frac{2}{45} \pi^{4}+\frac{9}{4} \zeta_{3}+\frac{1}{4}\right) a^{\left(n_{l}\right)}(\mu)\right. \\
& \left.-\frac{176}{3} \pi^{2} \log 2+\frac{325}{4} \zeta_{3}-\frac{2}{15} \pi^{4}+\frac{110}{3} \pi^{2}-\frac{73981}{108}\right] C_{A}^{2} \\
& +\left(-\frac{128}{3} \pi^{2} \log 2+16 \zeta_{3}+\frac{92}{3} \pi^{2}-\frac{613}{6}\right) C_{F} T_{F} n_{l}+\left(16 \pi^{2}-\frac{1013}{6}\right) C_{F} T_{F} \\
& +\left(\frac{64}{3} \pi^{2} \log 2+16 \zeta_{3}-\frac{32}{9} \pi^{2}+\frac{10816}{27}\right) C_{A} T_{F} n_{l} \\
& +\left(\frac{121}{18} a^{\left(n_{l}\right)}(\mu)-\frac{352}{9} \pi^{2}+\frac{11278}{27}\right) C_{A} T_{F} \\
& -\left(\frac{32}{9} \pi^{2}+\frac{1336}{27}\right) T_{F}^{2} n_{l}^{2}+\left(\frac{128}{9} \pi^{2}-\frac{3908}{27}\right) T_{F}^{2} n_{l}-\frac{20}{9} T_{F}^{2}, \\
& z_{30}=\left[-1792 a_{4}-\frac{224}{3} \log ^{4} 2+96 \pi^{2} \log ^{2} 2+\frac{3568}{3} \pi^{2} \log 2-20 \zeta_{5}\right. \\
& \left.+8 \pi^{2} \zeta_{3}-1256 \zeta_{3}-\frac{76}{15} \pi^{4}-\frac{4801}{9} \pi^{2}-\frac{3023}{12}\right] C_{F}^{2} \\
& +\left[-\frac{32}{3} a_{4}-\frac{4}{9} \log ^{4} 2-\frac{1448}{9} \pi^{2} \log ^{2} 2-\frac{2752}{9} \pi^{2} \log 2+580 \zeta_{5}\right. \\
& \left.-180 \pi^{2} \zeta_{3}-\frac{2312}{3} \zeta_{3}+\frac{6697}{270} \pi^{4}+\frac{2137}{9} \pi^{2}+\frac{24131}{72}\right] C_{F} C_{A} \\
& +\left[\left(-\frac{7}{6} \zeta_{5}-\frac{4}{9} \pi^{2} \zeta_{3}+\frac{13}{4} \zeta_{3}-\frac{17}{432} \pi^{4}+\frac{1}{4} \pi^{2}+\frac{13}{12}\right) a^{\left(n_{l}\right)}(\mu)\right. \\
& +\frac{1360}{3} a_{4}+\frac{170}{9} \log ^{4} 2+\frac{508}{9} \pi^{2} \log ^{2} 2-\frac{1300}{9} \pi^{2} \log 2-\frac{787}{2} \zeta_{5} \\
& \left.+\frac{340}{3} \pi^{2} \zeta_{3}+\frac{23311}{36} \zeta_{3}-\frac{20429}{2160} \pi^{4}-\frac{8705}{108} \pi^{2}-\frac{1656817}{1944}\right] C_{A}^{2} \\
& +\left[\frac{1024}{3} a_{4}+\frac{128}{9} \log ^{4} 2+\frac{256}{9} \pi^{2} \log ^{2} 2-\frac{1504}{9} \pi^{2} \log 2\right. \\
& \left.+\frac{1096}{3} \zeta_{3}-\frac{916}{135} \pi^{4}+\frac{904}{9} \pi^{2}+\frac{1120}{9}\right] C_{F} T_{F} n_{l} \\
& +\left[768 a_{4}+32 \log ^{4} 2-32 \pi^{2} \log ^{2} 2+\frac{1088}{9} \pi^{2} \log 2\right. \\
& \left.+\frac{466}{9} \zeta_{3}+\frac{124}{45} \pi^{4}-\frac{8848}{81} \pi^{2}-\frac{16811}{54}\right] C_{F} T_{F} \\
& +\left[-\frac{512}{3} a_{4}-\frac{64}{9} \log ^{4} 2-\frac{128}{9} \pi^{2} \log ^{2} 2+\frac{752}{9} \pi^{2} \log 2\right. \\
& \left.-\frac{280}{9} \zeta_{3}+\frac{152}{135} \pi^{4}+\frac{52}{3} \pi^{2}+\frac{111791}{243}\right] C_{A} T_{F} n_{l}
\end{aligned}
$$




$$
\begin{aligned}
& +\left[\left(\frac{8}{3} \zeta_{3}-\frac{2461}{108}\right) a^{\left(n_{l}\right)}(\mu)-512 a_{4}-\frac{64}{3} \log ^{4} 2+\frac{64}{3} \pi^{2} \log ^{2} 2+\frac{5120}{9} \pi^{2} \log 2\right. \\
& \left.\quad-60 \zeta_{5}+\frac{44}{3} \pi^{2} \zeta_{3}-\frac{2837}{9} \zeta_{3}-\frac{136}{45} \pi^{4}-\frac{36268}{81} \pi^{2}+\frac{100627}{81}\right] C_{A} T_{F} \\
& -\left(\frac{224}{9} \zeta_{3}+\frac{304}{27} \pi^{2}+\frac{11534}{243}\right) T_{F}^{2} n_{l}^{2}+\left(\frac{208}{9} \pi^{2}-\frac{18884}{81}\right) T_{F}^{2} n_{l} \\
& +\left(\frac{448}{3} \zeta_{3}+\frac{128}{45} \pi^{2}-\frac{16850}{81}\right) T_{F}^{2}
\end{aligned}
$$

(here $a_{4}=\operatorname{Li}_{4}(1 / 2)$ ). Gauge dependence first appears at three loops, as in $Z_{Q}^{\text {os }}$ [8]. The requirement of finiteness of the renormalized matching coefficient (5) at $\varepsilon \rightarrow 0$ has allowed the authors of [8] to extract $\tilde{Z}_{Q}$ from their result for $Z_{Q}^{\text {os }}$.

It would not be too difficult to take into account a lighter massive flavour, say, $m_{c} \neq 0$ in $b$-quark HQET. $\tilde{Z}_{Q}^{\text {os }}$ is no longer equal to 1 , but is known at three loops [14]; $Z_{Q}^{\text {os }}$ contains two scales, and is a non-trivial function of $m_{c} / m_{b}$ [15]. Both $\tilde{Z}_{Q}^{\text {os }}$ and $Z_{Q}^{\text {os }}$ have no smooth limit at $m_{c} \rightarrow 0$, but the discontinuity cancels in the ratio (4).

Now let's consider $z(\mu)$ in the large- $\beta_{0}$ limit (see Chapter 8 in [16] for a pedagogical introduction):

$$
z(\mu)=1+\int_{0}^{\beta} \frac{d \beta}{\beta}\left(\frac{\gamma(\beta)}{2 \beta}-\frac{\gamma_{0}}{2 \beta_{0}}\right)+\frac{1}{\beta_{0}} \int_{0}^{\infty} d u e^{-u / \beta} S(u)+\mathcal{O}\left(\frac{1}{\beta_{0}^{2}}\right),
$$

where $\beta=\beta_{0} \alpha_{s} /(4 \pi), \gamma=\gamma_{0} \alpha_{s} /(4 \pi)+\cdots$ (differences of $n_{l}$-flavour and $\left(n_{l}+1\right)$-flavour quantities can be neglected at the $1 / \beta_{0}$ order $)$. The difference of the QCD and HQET anomalous dimensions $\gamma=\gamma_{Q}-\tilde{\gamma}_{Q}$ and the Borel image $S(u)$ can be expressed as

$$
\gamma(\beta)=-2 \frac{\beta}{\beta_{0}} F(-\beta, 0), \quad S(u)=\frac{F(0, u)-F(0,0)}{u}
$$

where the function $F(\varepsilon, u)$ has been calculated in [1] (see also [16]):

$$
\begin{aligned}
F(\varepsilon, u)= & -2 C_{F}\left(\frac{\mu}{m}\right)^{2 u} e^{\gamma_{E} \varepsilon} \frac{\Gamma(1+u) \Gamma(1-2 u)}{\Gamma(3-u-\varepsilon)} D(\varepsilon)^{u / \varepsilon-1} \\
& \times(3-2 \varepsilon)(1-u)(1+u-\varepsilon) \\
D(\varepsilon)= & 6 e^{\gamma_{E} \varepsilon} \Gamma(1+\varepsilon) B(2-\varepsilon, 2-\varepsilon)=1+\frac{5}{3} \varepsilon+\cdots
\end{aligned}
$$

The anomalous dimension difference [1] is gauge invariant at this order:

$$
\gamma(\beta)=2 C_{F} \frac{\beta}{\beta_{0}} \frac{(1+\beta)\left(1+\frac{2}{3} \beta\right)}{B(2+\beta, 2+\beta) \Gamma(3+\beta) \Gamma(1-\beta)} ;
$$


the Borel image is [17,16]

$$
S(u)=-6 C_{F}\left[e^{(L+5 / 3) u} \frac{\Gamma(u) \Gamma(1-2 u)}{\Gamma(3-u)}\left(1-u^{2}\right)-\frac{1}{2 u}\right] .
$$

This Borel image has infrared renormalon poles at each positive half-integer $u$ and at $u=2$. Therefore, the integral in (88) is not well defined. Comparing its residue at the leading pole $u=1 / 2$ with the residue of the static-quark self-energy at its ultraviolet pole $u=1 / 2$ [18], we can express the renormalon ambiguity of $z(\mu)$ as

$$
\Delta z(\mu)=\frac{3}{2} \frac{\Delta \bar{\Lambda}}{m}
$$

( $\bar{\Lambda}$ is the ground-state meson residual energy). This ambiguity is compensated in physical matrix elements by ultraviolet renormalon ambiguities in the leading $1 / m$ correction (matrix elements of both local and bilocal dimension-5/2 operators), see [17].

The matching coefficient is gauge invariant at the order $1 / \beta_{0}$. Expanding $\gamma(\beta)$ and $S(u)$ and integrating, we obtain

$$
\begin{gathered}
z(\mu)=1-C_{F} \frac{\alpha_{s}(\mu)}{4 \pi}\left\{3 L+4+\left[\frac{3}{2} L^{2}+\frac{19}{2} L+\pi^{2}+\frac{113}{8}\right] \frac{\beta_{0} \alpha_{s}(\mu)}{4 \pi}\right. \\
+\left[L^{3}+\frac{19}{2} L^{2}+\left(2 \pi^{2}+\frac{167}{6}\right) L+14 \zeta_{3}+\frac{19}{3} \pi^{2}+\frac{5767}{216}\right]\left(\frac{\beta_{0} \alpha_{s}(\mu)}{4 \pi}\right)^{2} \\
+\left[\frac{3}{4} L^{4}+\frac{19}{2} L^{3}+\left(3 \pi^{2}+\frac{167}{4}\right) L^{2}+\left(36 \zeta_{3}+19 \pi^{2}+\frac{2903}{36}\right) L\right. \\
\left.\left.+\frac{71}{40} \pi^{4}+\frac{467}{4} \zeta_{3}+\frac{167}{6} \pi^{2}+\frac{103933}{1728}\right]\left(\frac{\beta_{0} \alpha_{s}(\mu)}{4 \pi}\right)^{3}+\cdots\right\}
\end{gathered}
$$

Thus we have confirmed the contributions with the highest power of $n_{l}$ in each term in (7), and predicted such a contribution at $\alpha_{s}^{4}$.

Numerically, the matching coefficient (7) in the Landau gauge at $\mu=m$ and $n_{l}=4$ can be written as

$$
\begin{aligned}
z(m)= & 1-\frac{4}{3} \frac{\alpha_{s}^{(4)}(m)}{\pi}-\left(1.9996 \beta_{0}-4.5421\right)\left(\frac{\alpha_{s}^{(4)}(m)}{\pi}\right)^{2} \\
& -\left(2.2091 \beta_{0}^{2}+5.1153 \beta_{0}-61.5397\right)\left(\frac{\alpha_{s}^{(4)}(m)}{\pi}\right)^{3} \\
& -\left(3.3755 \beta_{0}^{3}+\cdots\right)\left(\frac{\alpha_{s}^{(4)}(m)}{\pi}\right)^{4}+\cdots
\end{aligned}
$$




$$
\begin{aligned}
= & -\frac{4}{3} \frac{\alpha_{s}^{(4)}(m)}{\pi}-(16.6629-4.5421)\left(\frac{\alpha_{s}^{(4)}(m)}{\pi}\right)^{2} \\
& -(153.4076+42.6271-61.5397)\left(\frac{\alpha_{s}^{(4)}(m)}{\pi}\right)^{3} \\
& -(1953.4013+\cdots)\left(\frac{\alpha_{s}^{(4)}(m)}{\pi}\right)^{4}+\cdots \\
= & 1-\frac{4}{3} \frac{\alpha_{s}^{(4)}(m)}{\pi}-12.1208\left(\frac{\alpha_{s}^{(4)}(m)}{\pi}\right)^{2}-134.4950\left(\frac{\alpha_{s}^{(4)}(m)}{\pi}\right)^{3} \\
& -(1953.4013+\cdots)\left(\frac{\alpha_{s}^{(4)}(m)}{\pi}\right)^{4}+\cdots
\end{aligned}
$$

( $\beta_{0}$ is for $n_{l}=4$ flavours). Naive nonabelianization [1] works rather well at two and three loops (in the latter case the $\mathcal{O}\left(\beta_{0}\right)$ and $\mathcal{O}(1)$ terms partially compensate each other, similarly to [2]). Therefore, we can expect that the estimate of the $\alpha_{s}^{4}$ term is also reasonably good. Numerical convergence of the series is very poor; this is related to the infrared renormalon at $u=1 / 2$.

Now let us consider the relation between the $\overline{\mathrm{MS}}$ renormalized electron field in QED and the Bloch-Nordsieck electron field [19]. The bare matching coefficient $z_{0}=Z_{\psi}^{\text {os }}$ is gauge invariant to all orders [20,21,8]. In the BlochNordsieck model, due to exponentiation [22], $\log \tilde{Z}_{\psi}=\left(3-a^{(0)}\right) \alpha^{(0)} /(4 \pi \varepsilon)$ (where the 0-flavour $\alpha^{(0)}$ is equal to the on-shell $\alpha \approx 1 / 137$ ). In the full QED, $\log Z_{\psi}=-a^{(1)} \alpha^{(1)} /(4 \pi \varepsilon)+$ (gauge-invariant higher terms), see the Appendix. The gauge dependence cancels in $\log \left(\tilde{Z}_{\psi} / Z_{\psi}\right)$ because of the QED decoupling relation $a^{(1)} \alpha^{(1)}=a^{(0)} \alpha^{(0)}$. Therefore, the renormalized matching coefficient $z(\mu)$ in QED is gauge invariant to all orders. We obtain

$$
\begin{aligned}
z(\mu)=1 & -(3 L+4) \frac{\alpha}{4 \pi} \\
+ & \left(\frac{9}{2} L^{2}+\frac{31}{2} L+16 \pi^{2} \log 2-24 \zeta_{3}-\frac{55}{3} \pi^{2}+\frac{5957}{72}\right)\left(\frac{\alpha}{4 \pi}\right)^{2} \\
- & {\left[\frac{9}{2} L^{3}+\frac{143}{6} L^{2}+\left(48 \pi^{2} \log 2-72 \zeta_{3}-55 \pi^{2}+\frac{19363}{72}\right) L\right.} \\
& +1024 a_{4}+\frac{128}{3} \log ^{4} 2-64 \pi^{2} \log ^{2} 2-\frac{11792}{9} \pi^{2} \log 2+20 \zeta_{5} \\
& \left.-8 \pi^{2} \zeta_{3}+\frac{9494}{9} \zeta_{3}+\frac{104}{45} \pi^{4}+\frac{259133}{405} \pi^{2}+\frac{249887}{324}\right]\left(\frac{\alpha}{4 \pi}\right)^{3}+\cdots
\end{aligned}
$$

In conclusion: we have derived the QCD/HQET matching coefficient for the heavy-quark field with three-loop accuracy (7), and the all-orders result in the large- $\beta_{0}$ limit. The corresponding QED coefficient (16) is gauge invariant. 
Acknowledgements. I am grateful to K.G. Chetyrkin and M. Steinhauser for useful discussions and hospitality in Karlsruhe. The work was supported by DFG through SFB/TR9.

\section{Appendix. Gauge dependence of $Z_{\psi}$ and $\gamma_{\psi}$ in QED.}

The electron propagator $S(x)$ is related to the Landau-gauge propagator $S_{L}(x)$ $\left(a_{0}=0\right)$ by the formula [20]

$$
S(x)=S_{L}(x) e^{-i e_{0}^{2}(\Delta(x)-\Delta(0))},
$$

where $\Delta(x)$ is the Fourier image of $\Delta(k)=a_{0} /\left(k^{2}\right)^{2}$, and $\Delta(0)=0$ in dimensional regularization. The electron field renormalization does not depend on its mass. For simplicity, we shall consider the massless electron, whose propagator has a single Dirac structure:

$$
S(x)=S_{0}(x) e^{\sigma(x)}
$$

where $S_{0}(x)$ is the $d$-dimensional free massless electron propagator. Then

$$
\sigma(x)=\sigma_{L}(x)+a_{0} \frac{e_{0}^{2}}{(4 \pi)^{d / 2}}\left(\frac{-x^{2}}{4}\right)^{\varepsilon} \Gamma(-\varepsilon),
$$

where the Landau-gauge $\sigma_{L}$ starts from $e_{0}^{4}$. Re-expressing $\sigma$ via renormalized quantities we have

$$
\sigma(x)=\sigma_{L}(x)+a(\mu) \frac{\alpha(\mu)}{4 \pi}\left(\frac{-\mu^{2} x^{2}}{4}\right)^{\varepsilon} e^{\gamma_{E} \varepsilon} \Gamma(-\varepsilon) .
$$

This must be equal to $\log Z_{\psi}+\sigma_{r}$, where $\log Z_{\psi}(\alpha(\mu), a(\mu))$ contains only negative powers of $\varepsilon$, and the renormalized $\sigma_{r}$ - only non-negative. Therefore,

$$
\log Z_{\psi}(\alpha, a)=\log Z_{L}(\alpha)-a \frac{\alpha}{4 \pi \varepsilon} .
$$

In QED $d \log (a(\mu) \alpha(\mu)) / d \log \mu=-2 \varepsilon$ exactly, and 1

$$
\gamma_{\psi}(\alpha, a)=2 a \frac{\alpha}{4 \pi}+\gamma_{L}(\alpha)
$$

where the Landau-gauge $\gamma_{L}(\alpha)$ starts from $\alpha^{2}$.

$\overline{1}$ I was informed by D.J. Broadhurst and D.V. Shirkov that this result has been proved in some Russian article in the second half of 50s. I am grateful to them for discussing this question; unfortunately, I was unable to find this article. 


\section{References}

[1] D.J. Broadhurst, A.G. Grozin, Phys. Rev. D 52 (1995) 4082 arXiv:hep-ph/9410240.

[2] S. Bekavac, A.G. Grozin, P. Marquard, J.H. Piclum, D. Seidel, M. Steinhauser, Nucl. Phys. B 833 (2010) 46 [arXiv:0911.3356 [hep-ph]].

[3] C.L.Y. Lee, Preprint CALT-68-1663, Caltech (1991); revised (1997).

[4] J.G. Körner, G. Thompson, Phys. Lett. B 264 (1991) 185;

S. Balk, J.G. Körner, D. Pirjol, Nucl. Phys. B 428 (1994) 499 arXiv:hep-ph/9307230.

[5] T. Mannel, W. Roberts, Z. Ryzak, Nucl. Phys. B 368 (1992) 204.

[6] M. Neubert, Phys. Rep. 245 (1994) 259 arXiv:hep-ph/9306320].

[7] M.E. Luke, A.V. Manohar, Phys. Lett. B 286 (1992) 348.

[8] K. Melnikov, T. van Ritbergen, Nucl. Phys. B 591 (2000) 515 arXiv:hep-ph/0005131.

[9] K.G. Chetyrkin, A.G. Grozin, Nucl. Phys. B 666 (2003) 289 arXiv:hep-ph/0303113.

[10] O.V. Tarasov, Preprint JINR P2-82-900, Dubna (1982).

[11] S.A. Larin, J.A.M. Vermaseren, Phys. Lett. B 303 (1993) 334 arXiv:hep-ph/9302208.

[12] K.G. Chetyrkin, B.A. Kniehl, M. Steinhauser, Nucl. Phys. B 510 (1998) 61 arXiv:hep-ph/9708255.

[13] A.G. Grozin, P. Marquard, J.H. Piclum, M. Steinhauser, Nucl. Phys. B 789 (2008) 277 [arXiv:0707.1388 [hep-ph]].

[14] A.G. Grozin, A.V. Smirnov, V.A. Smirnov, JHEP 11 (2006) 022 arXiv:hep-ph/0609280.

[15] S. Bekavac, A.G. Grozin, D. Seidel, M. Steinhauser, JHEP 10 (2007) 006 arXiv:0708.1729 [hep-ph]].

[16] A.G. Grozin, Heavy Quark Effective Theory, Springer Tracts in Modern Physics 201, Springer (2004).

[17] M. Neubert, C.T. Sachrajda, Nucl. Phys. B 438 (1995) 235 arXiv:hep-ph/9407394.

[18] M. Beneke, V.M. Braun, Nucl. Phys. B 426 (1994) 301 arXiv:hep-ph/9402364.

[19] F. Bloch, A. Nordsieck, Phys. Rev. 52 (1937) 54. 
[20] L.D. Landau, I.M. Khalatnikov, Sov. Phys. JETP 2 (1956) 69.

[21] K. Johnson, B. Zumino, Phys. Rev. Lett. 3 (1959) 351.

[22] D.R. Yennie, S.C. Frautschi, H. Suura, Ann. Phys. 13 (1961) 379. 